\title{
Identification of Textile Materials Properties in "Body-Clothes" Scanned Systems
}

\author{
Iulia S. ZVEREVA*a, Victor E. KUZMICHEV ${ }^{a}$, DominiqueC. ADOLPHE ${ }^{b}$, L. SCHACHER ${ }^{b}$ \\ ${ }^{a}$ Ivanovo State Textile Academy, Department of Garment Design, Ivanovo, Russia \\ ${ }^{b}$ University of Haute Alsace - LPMT - ENSISA, Mulhouse, France
}

\begin{abstract}
The important problem of the realistic 3D design is to reflect the features of different textile materials and to create the surface of virtual clothes from textile shell. In our research, the traditional indexes of physical and mechanical properties of fabrics has been measured and analyzed and some new indexes for describing the "shell properties" measured on the real clothes surfaces have been developed. Proposed method and mathematical equations allow to identify the indexes of fabric in the "body-clothes" scanned systems in order to design in a more realistic manner the trousers in 3D.
\end{abstract}

Keywords: body scanning, realistic surface, wrinkles, fabric properties, "body-trousers" system, identification.

\section{Introduction}

The main goal of research in garment technology is to provide realistic 3D virtual design of the «bodyclothes» system. Modern technical devises, body scanners and software allow generating, designing and modeling 3D models of typical human body and clothes with a smooth surface [1,2].

It's necessary to know the shaping abilities of the flat clothes patterns made of different materials after transformation into three dimensional shells. Currently, one of the most important problems is to reflect the physical and mechanical properties of different textile materials and to create the more realistic surface of virtual clothes made from these textile shells.

Fathy and Kenkare $[3,4]$ studied influence of fabric properties on drape ability only for folds simulation purpose. Fabric draping and flat pattern parameters of the clothes both influence air gaps values existing between the body and the shell, the folds and the wrinkles which appear on the surface.

Modern software for designers is proposed by Wang and Cheng [5] to create folds and wrinkles along ef the bottom like skirts and draped details. It is necessary to know draping particularities of the fabric and wrinkles locations on the other areas of clothes surface.

Juan et al. [6] developed sketch-based method to generate arbitrary wrinkles. Such methods are very useful for animation and 3D visualization but cannot provide realistic view of virtual clothes and its adequate transformation to the flat patterns.

To improve the realistic 3D clothes design made of different fabrics for ready-to-wear needs, it is necessary to get additional knowledge and information. Today, only body scanning technology can provides realistic 3D digital images and allows unlimited retrieval of precise linear, two-, and threedimensional measurements of the object [7]. After analyzing the cross-sections, the air gaps values in different areas can be obtained and used to create new 3D images. That is why body scanning is used in research of 3D clothes shaping with different ease values to investigate the 3D "body-clothes" systems in static and dynamic situation [8, 9].

This work presents the research results of textile fabric properties which influencing to the 3D clothes surface shaping using body scanning technology.

The difficult relief of clothes surface is formed under the influence of (1) body morphology and its position, (2) sizes and lines configuration of the clothes pattern details, (3) shape generation elements such as darts, (4) clothes construction, (5) indexes of the physical and mechanical properties of the textile materials.

Figure 1 shows the structure of the "body-clothes" realistic design using database obtained after body scanning.

“julia-zve@mail.ru; +7 9621581523 


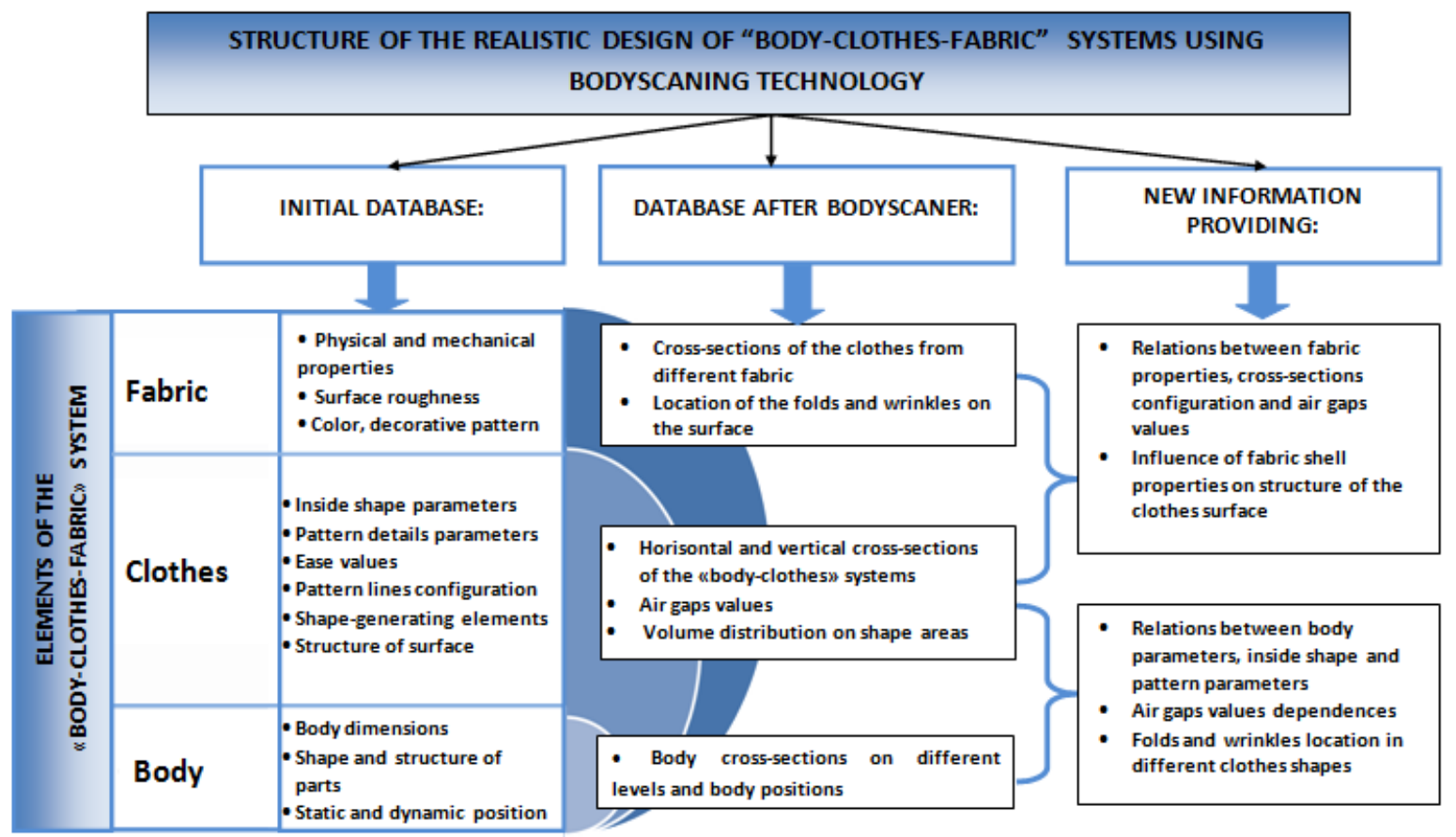

Fig. 1 Structure of the "body-clothes" realistic design.

The object of our research is the design of the trousers with different 3D shapes made from different textile fabrics. The previous works in this area made by Ashdown, Sunyoon and Petrova, researched the relations between the ease values and the body sizes [10] and also proposed the approach to modeling 3D virtual trousers using the air gaps values [11].

The appearance and structure of the real surface, folds and wrinkles are influenced by trousers pattern parameters and fabric properties.

The main aim of our research is to analyze traditional indexes of the physical and mechanical properties of textile materials samples (PMP) and to develop some new indexes for describing shell properties (SP) of real trousers surface. Two factors of trousers surface shaping were investigated: first is ease values of the pattern blocks, second is the physical and mechanical properties of fabric. Study of these factors will allow to establish the relationships between the fabric properties and the wrinkle coefficient of realistic trousers shapes.

\section{Methods}

Research and experimental plan included the following steps:

1. Choosing of 10 samples of textile fabrics with different properties and the testing of physical and mechanical properties by means of Kawabata Evaluation System.

2. Creating trousers patterns with different parameters and ease values (ease to hip girth $E_{\mathrm{hg}}=3 \ldots 11.5 \mathrm{~cm}$, ease to thigh girth $E_{\mathrm{tg}}=12 \ldots 22.8 \mathrm{~cm}$ ).

3. Sewing of the men trousers from chosen fabrics.

4. Scanning of the "typical men 182-100-88 - trousers" (to be explained) using the TELMAT body scanner.

5. Obtaining and combining the cross-sections from the "body-trousers" systems on hip and thigh levels [12].

6. Developing the new indexes for describing trousers surface.

7. Calculating of the new wrinkle coefficients.

8. Performing correlation analysis of relations between wrinkle coefficients, fabric properties and ease values.

9. Obtaining the mathematical equations describing the new indexes.

10. Developing the new method for identification of fabric properties and designing realistic surface of the "body-trousers-fabric" systems. 


\subsection{Investigation of the standard physical and mechanical properties of the fabric samples}

Kawabata Evaluation System (KES) was used for testing physical and mechanical properties of the fabric samples. Experimental tests were conducted at the University of Haute Alsace - ENSISA LPMT (Mulhouse, France).

Several properties of the 10 samples of fabric were measured: tensile (stretch), shear stiffness (drape), bending rigidity (flexing), compression (thickness, softness), and surface friction and roughness [13].

Using Shear/Tensile Strength Tester KES F-1 the following indexes were measured:

1 G - shear stiffness, $\mathrm{gf} / \mathrm{cm}$. degree, is the degree with which yarns move, resulting in soft / pliable to stiff / rigid structures.

2 EMT - extensibility or stretch, percent strain at maximum applied force: completely elastic $(100 \%)$ to inelastic $(0 \%)$; higher value indicates greater stretch.

3 RT - tensile resilience, percent recovery from stretch when the applied force is removed. Higher values indicate greater recovery from having been stretched.

Using Pure Bending Tester KES FB-2 one index was measured:

$B$ - bending rigidity per unit fabric width, $\mathrm{gf} . \mathrm{cm}^{2} / \mathrm{cm}$. Higher $B$ value indicates greater stiffness / resistance to bending motions.

Using Compression Tester KES FB-3 the next indexes were measured:

1. $\quad \mathbf{R C}$ - compressional resilience, percent recovery or regain in thickness, when the force is removed. Higher value indicates a greater recovery from being compressed.

2. To - thickness; measured at $0.5 \mathrm{gf} / \mathrm{cm}^{2}$.

Using Surface Tester KES F-4 were measured indexes:

1. MIU - coefficient of friction, 0 to 1 with higher value corresponding to greater friction or resistance and drag.

2. SMD - geometric roughness, micron; higher values corresponds to geometrically rougher surface

Results of the tests with variable coefficients are shown in the table 1.

Table 1 - Results of the testing physical and mechanical properties of the fabric samples.

\begin{tabular}{|c|c|c|c|c|c|c|c|c|}
\hline \multirow{2}{*}{ Property } & \multirow{2}{*}{ Index } & \multirow{2}{*}{ Metages } & \multicolumn{3}{|c|}{ WARP } & \multicolumn{3}{|c|}{ WEFT } \\
\hline & & & $\min$ & $\max$ & V (\%) & $\min$ & $\max$ & $\mathrm{V}(\%)$ \\
\hline \multirow{2}{*}{ Tensile } & EMT & $\%$ & 7.57 & 33.91 & 76.95 & 16.48 & 94.66 & 59.03 \\
\hline & RT & $\%$ & 23.08 & 50.86 & 24 & 14.26 & 46.95 & 25.11 \\
\hline Bending & B & gf. $\mathrm{cm}^{2} / \mathrm{cm}$ & 0.04 & 0.42 & 54.4 & 0.06 & 0.7 & 70.6 \\
\hline Shearing & G & gf/cm.deg & 0.41 & 5.15 & 73 & 0.44 & 4.36 & 64.4 \\
\hline \multirow{3}{*}{ Surface } & MIU & - & 0.001 & 0.31 & 64.01 & 0.12 & 0.32 & 29.47 \\
\hline & SMD & - & 0.01 & 3.11 & 64.36 & 1.26 & 3.27 & 29.47 \\
\hline & & & \multicolumn{2}{|c|}{$\frac{1}{\min }$} & \multicolumn{2}{|c|}{$\max$} & \multicolumn{2}{|c|}{$\mathrm{V}(\%)$} \\
\hline Compression & $\mathrm{RC}$ & $\%$ & \multicolumn{2}{|c|}{43.31} & \multicolumn{2}{|c|}{74.44} & \multicolumn{2}{|c|}{17} \\
\hline Thickness & To & $\mathrm{mm}$ & \multirow{2}{*}{\multicolumn{2}{|c|}{$\begin{array}{l}0.35 \\
116\end{array}$}} & \multicolumn{2}{|c|}{1.71} & \multicolumn{2}{|c|}{$\frac{49.58}{25.76}$} \\
\hline Weight & W & g.m2 & & & \multicolumn{2}{|c|}{342} & \multicolumn{2}{|c|}{25.76} \\
\hline
\end{tabular}

Table 1 shows that the indexes of properties are varied in wide ranges, that allows to study their influence on trousers shell surface more objectively. Comparing parameters of the three-dimensional "body-trousers" systems from different materials at constancy of pattern parameters will allow estimating the influence of the fabric properties on the wrinkles location.

\subsection{Investigation of three dimensional "body-trousers-fabric" systems}

Trousers with different shapes and made from 10 fabrics were used for this study. By means of the TELMAT body scanner (France) trousers were scanned on the typical 182-100-88 (to be explained) men body and the "body-trousers" systems were obtained (fig.2). 


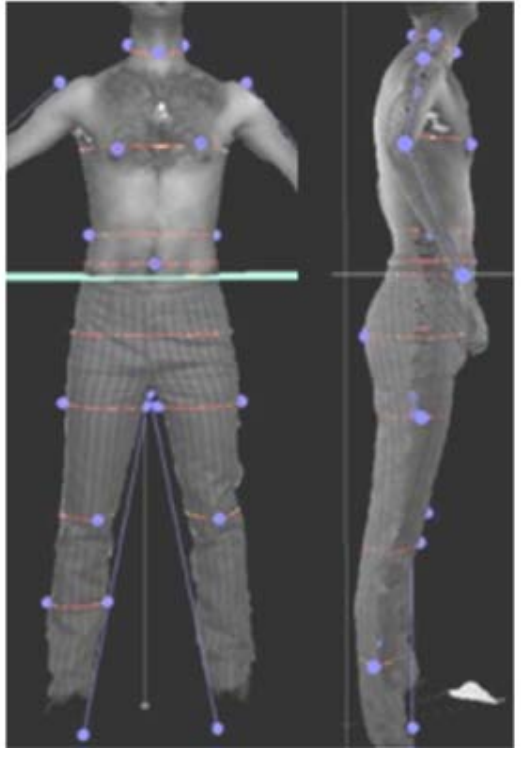

a

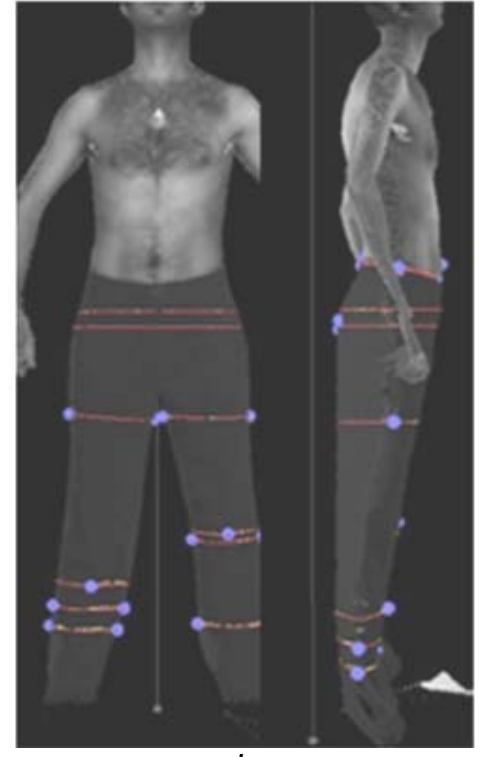

$b$

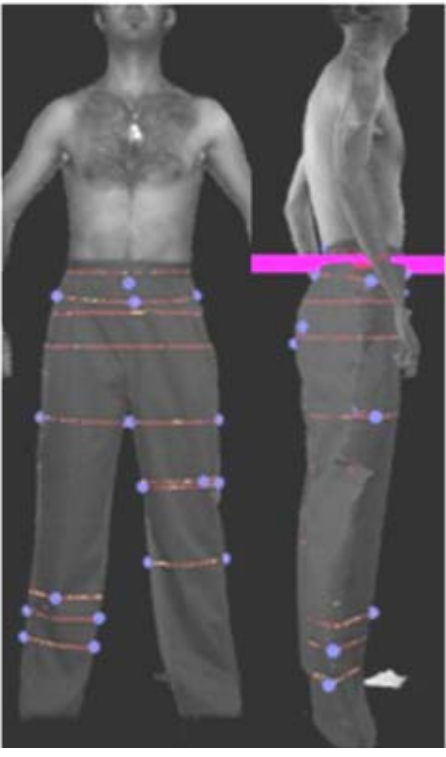

C

Fig. 2. Images of $3 D$ "body-trousers" systems made from different fabrics $a$ - close fitting, $b$ - middle fitting, $c$ - loose fitting.

After the parameterization of real surface, the specific cross-sections at different levels where trousers shapes and PMP indexes influenced on the surface smoothness, have been obtained. Horizontal cross-sections at the hip and thigh levels of body and trousers were combined in the geometrical center and separate in several sectors as shown on fig. 3.

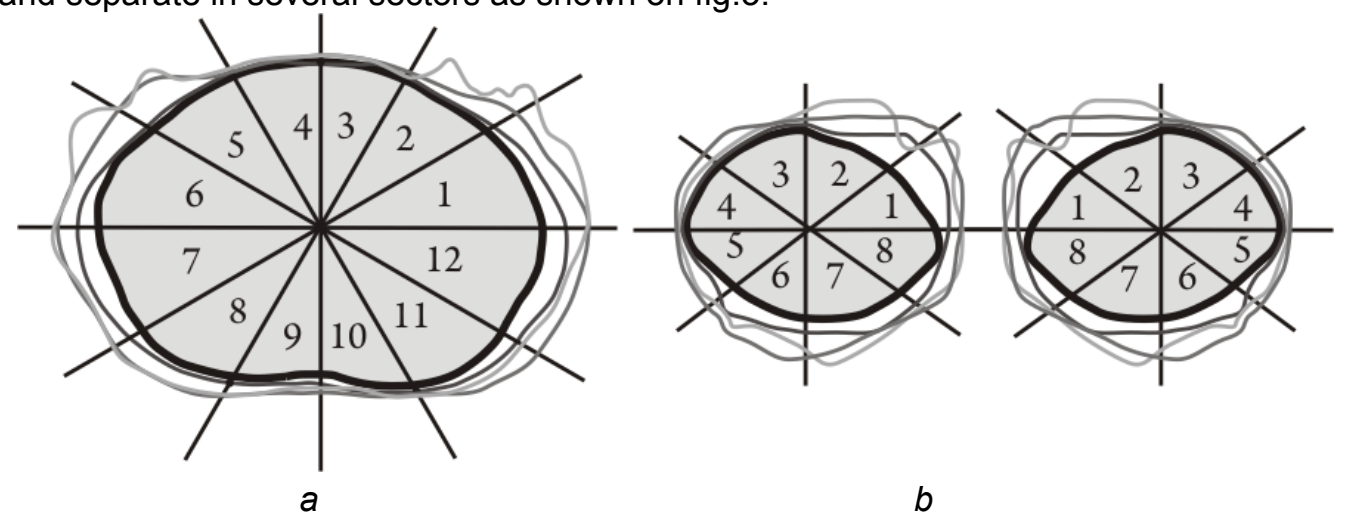

Fig. 3. Cross-sections on the hip (a) and thigh (b) levels with the sectors scheme.

The cross-sections of "body-trousers" systems with different pattern parameters and fabric indexes properties were thus got. Configuration of cross-sections differs in each sector and to study the factors influencing the surface it is necessary to describe the wrinkles by new indexes.

\subsection{Developing the new indexes of fabric shell properties}

It is difficult to describe realistic plicate surface of the trousers shell with simple geometrical parameters, because of its variability and wrinkle structure. Traditional PMP (to be explained) of the fabric and geometrical parameters of clothes shape influence on the surface structure in complex and it is not possible to describe the shell by the universal index of properties.

Thus to study fabric surface in 3D, a special index for shell properties (SP) was used, that characterize the fabric ability to form the shell around the body with the folds and wrinkles.

To describe the SP properties, the special wrinkle parameters (fig.4) and the wrinkle coefficient calculated by the following equation:

$$
C w_{\mathrm{i}}=100\left(I f_{\mathrm{i}}-I b_{\mathrm{i}}\right) / I b_{\mathrm{i}}
$$

where are $C w_{i}$ - wrinkle coefficient for $i$ - section, $I b_{\mathrm{i}}$ - the full length of $i$ - section measured on the human body, $I f_{\mathrm{i}}$ - the full length of $i$ - section fabric shell measured on the trousers. 


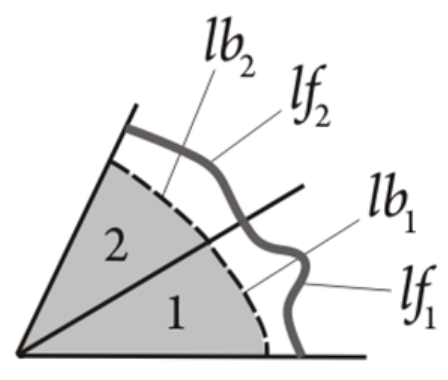

$I b_{\mathrm{i}}-$ length of the body shell,
$I f_{\mathrm{i}}-$ length of the fabric shell.

Fig. 4. Scheme of the wrinkle parameters measuring.

The index $C w_{i}$ of fabric wrinkle of the required area allow to estimate the increasing of the fabric surface length comparing to the body surface length in this area.

Using equation (1) it is possible to calculate the length of the clothes shell and to design wrinkles according to it.

Database of wrinkle coefficients $C w_{i}$ was obtained for "body-trousers" studied systems issued from fabrics with different properties.

Values of the wrinkle coefficients with variable coefficients for fabrics with different bending rigidity $B$ is shown in the table 2. To compare the results, all tested fabrics were separate in three groups.

It was established that the wrinkles of the shell depends on fabric properties and area of the trousers shell. Under influence of properties surpluses of the surface shell length (ease value of clothes) can be formed in folds and wrinkles in one or another sector. For prognostication and control of this process it is necessary to obtain mathematical equation between the wrinkle coefficient $\mathrm{Cw}_{i}$ and indexes of fabric properties.

Table 2. Values of the wrinkle coefficients $C w_{i}$ for trousers shell.

\begin{tabular}{|c|c|c|c|c|c|c|c|c|c|}
\hline & \multicolumn{9}{|c|}{$\begin{array}{c}\text { Values of the wrinkle coefficients } C w_{i} \text { and } \text { variable coefficients for fabrics with different bending } \\
\text { rigidity } B\end{array}$} \\
\hline$B$, gf.cm $2 / \mathrm{cm}$ & \multicolumn{3}{|c|}{$0.04-0.15$} & \multicolumn{3}{|c|}{$0.15-0.23$} & \multicolumn{3}{|c|}{$0.23-0.42$} \\
\hline \multirow{2}{*}{ Sector } & \multicolumn{9}{|c|}{ Hip level } \\
\hline & $\min$ & $\max$ & V (\%) & $\min$ & $\max$ & $\mathrm{V}(\%)$ & $\min$ & $\max$ & $\mathrm{V}(\%)$ \\
\hline 1 & 2.61 & 16.67 & 49.8 & 0.80 & 15.10 & 69.0 & 6.53 & 15.16 & 27.4 \\
\hline 2 & 2.45 & 18.33 & 54.2 & 3.53 & 26.33 & 60.5 & 1.16 & 17.92 & 76.8 \\
\hline 3 & 1.71 & 13.91 & 45.5 & 2.74 & 12.50 & 40.8 & 0.00 & 27.12 & 98.9 \\
\hline 4 & 0.00 & 13.76 & 78.9 & 4.24 & 12.66 & 36.6 & 0.85 & 17.25 & 70.1 \\
\hline 5 & 0.64 & 17.87 & 44.0 & 6.51 & 33.33 & 53.2 & 0.38 & 21.60 & 56.6 \\
\hline 6 & 3.55 & 11.03 & 28.1 & 0.97 & 6.90 & 51.5 & 1.92 & 14.22 & 47.1 \\
\hline 7 & 4.35 & 36.42 & 57.9 & 0.78 & 18.19 & 54.8 & 4.91 & 20.59 & 48.2 \\
\hline 8 & 4.43 & 21.02 & 41.2 & 5.57 & 15.47 & 31.5 & 4.75 & 11.13 & 23.4 \\
\hline 9 & 4.23 & 6.62 & 10.3 & 6.83 & 10.82 & 14.3 & 3.54 & 18.47 & 71.2 \\
\hline 10 & 0.60 & 7.81 & 44.2 & 5.10 & 13.94 & 24.9 & 0.80 & 16.93 & 92.1 \\
\hline 11 & 2.99 & 43.01 & 61.0 & 0.25 & 38.07 & 98.1 & 1.49 & 27.19 & 74.5 \\
\hline 12 & 7.72 & 25.75 & 37.8 & 0.11 & 21.75 & 69.9 & 5.76 & 20.60 & 34.7 \\
\hline \multirow{2}{*}{ Sector } & \multicolumn{9}{|c|}{ Tight level } \\
\hline & $\min$ & $\max$ & V (\%) & $\min$ & $\max$ & V (\%) & $\min$ & $\max$ & V (\%) \\
\hline 1 & 4.78 & 24.27 & 36.9 & 8.44 & 28.78 & 15.5 & 2.46 & 33.69 & 59.1 \\
\hline 2 & 12.05 & 59.33 & 59.1 & 16.24 & 37.19 & 34.5 & 12.19 & 38.49 & 41.9 \\
\hline 3 & 2.24 & 25.45 & 58.1 & 6.59 & 20.54 & 48.9 & 9.96 & 25.52 & 32.4 \\
\hline 4 & 1.13 & 40.26 & 89.4 & 7.18 & 19.50 & 32.3 & 9.10 & 42.05 & 62.7 \\
\hline 5 & 0.51 & 17.23 & 63.3 & 5.66 & 18.30 & 40.7 & 3.08 & 32.77 & 66.2 \\
\hline 6 & 0.82 & 51.44 & 78.4 & 11.81 & 28.34 & 28.5 & 1.13 & 25.09 & 57.9 \\
\hline 7 & 2.82 & 30.13 & 59.9 & 10.00 & 57.44 & 67.8 & 10.00 & 44.26 & 53.7 \\
\hline 8 & 13.23 & 28.81 & 24.1 & 3.59 & 14.50 & 51.8 & 0.67 & 19.19 & 47.3 \\
\hline
\end{tabular}




\section{Results}

After the data analyzes, the mathematical relations were established between the wrinkle coefficient $C w_{i}$ and PMP indexes for the trousers with different ease to hip girth $E_{\mathrm{hg}}$.

Correlation analysis allowed establishing that the bending rigidity $B$ and the shear stiffness $G$ influence on the $C w_{i}$ over all other properties $(R=0.55 \ldots 0.82)$.

$B$ and $G$ indexes characterize shell properties of the fabric and can be measured by Kawabata system (fig.5a). Histograms for the 10 tested samples shown that the Bending rigidity and Shear stiffness are not interdependent (fig.5b) and that it is necessary to include both indexes into the regression equations.

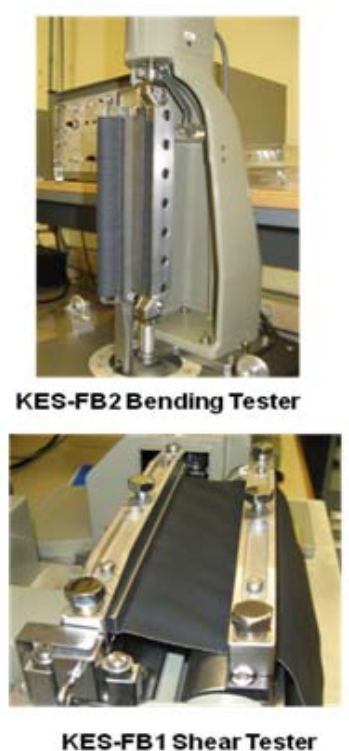

a
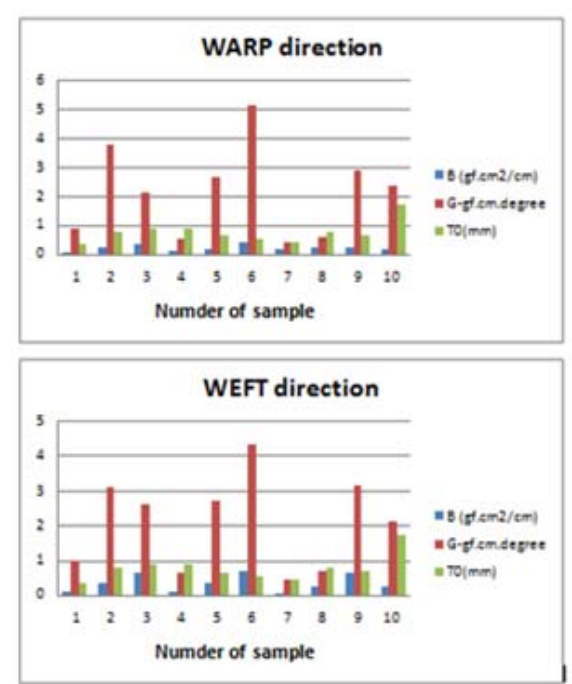

b

Fig. 5. Kawabata technical devises for testing bending rigidity $B$ and shear stiffness $G(a)$. Histograms of indexes values for 10 tested samples (b).

For 182-100-88 men body, mathematical relations for the trousers wrinkle coefficient $C w_{i}$ on the hip and thigh levels are shown in the table 3.

The location of the wrinkles is not the same with the different fabrics and trousers shapes, but there are sectors of surface which are influenced by fabric properties most of all and in which wrinkle coefficient $\mathrm{Cw}_{\mathrm{i}}$ can be changed. For example, on the hip level it is the sectors number 1-6 of the front part of the trousers. On the thigh level it is sectors number 3-6 of the inside part of the trousers.

Table 3. Mathematical equations for the calculation of the wrinkle coefficient $\mathrm{Cw}_{i .}$

\begin{tabular}{|c|c|c|c|}
\hline \multirow{2}{*}{$\mathrm{Cw}_{\mathrm{i}}$} & \multirow{2}{*}{ Mathematical equations } & \multicolumn{2}{|c|}{ Statistics } \\
\hline & & $F$ & $R$ \\
\hline 1 & 2 & 3 & 4 \\
\hline \multicolumn{4}{|c|}{ Hip girth level } \\
\hline $\begin{array}{l}\mathrm{Cw}_{2} . \\
\mathrm{Cw} w_{5}\end{array}$ & $4.03 E_{\mathrm{hg}}+3.47 \mathrm{G}_{\mathrm{wr}}-0.07 \mathrm{G}_{\mathrm{wf}}-16.94$ & 6.85 & 0.69 \\
\hline $\begin{array}{l}\mathrm{CW}_{3} . \\
\mathrm{CW}_{4}\end{array}$ & $-0.87 E_{\mathrm{hg}}-10.87 B_{\mathrm{wr}}-10.99 B_{\mathrm{wf}}+4.12 \mathrm{G}_{\mathrm{wr}}+0.33 \mathrm{G}_{\mathrm{wf}}+8.78$ & 3.80 & 0.70 \\
\hline $\begin{array}{l}C w_{1} . \\
C w_{6}\end{array}$ & $1.52 E_{\mathrm{hg}}+7.63 B_{\mathrm{wr}}-2.2 B_{\mathrm{wf}}+8.92 \mathrm{G}_{\mathrm{wr}}-8.2 \mathrm{G}_{\mathrm{wf}}-3.65$ & 4.38 & 0.72 \\
\hline \multicolumn{4}{|c|}{ Thigh girth level } \\
\hline$C w_{3}$ & $-31.87 B_{w r}+58.81 B_{w f}+6.45 G_{w r}-8.74 G_{w f}+4.25$ & 2.96 & 0.55 \\
\hline$C w_{4}$ & $25.19 B_{\mathrm{wr}}+7.54$ & 4.45 & 0.77 \\
\hline$C w_{5}$ & $18.49 B_{w r}+78.18 B_{w f}+2.99 G_{w r}-2.59 G_{w f}+0.19$ & 3.34 & 0.56 \\
\hline$C w_{6}$ & $-61.4 B_{\mathrm{wr}}+12.21$ & 6.04 & 0.82 \\
\hline $\begin{array}{l}\text { Where } \\
B_{\mathrm{wr}} \text { и } B \\
\text { directio }\end{array}$ & $\begin{array}{l}\text { ing to half hip girth. } \mathrm{cm} \text {; } \\
\text { deg. }\end{array}$ & s in wa & \\
\hline
\end{tabular}

Note: crucial values of $R=0.29 . F=2.63$ for confiding probability $95 \%$ and $n=32$. 
In order to check the equations, the wrinkle coefficients $\mathrm{Cw}_{\mathrm{i}}$ were calculated for three typical trousers fabrics (tab. 4). After, the real "body-trousers" systems were analyzed using the proposed method. Theoretical results and real values of the coefficients were compared.

Table 4. Wrinkle coefficients values for three types of fabric.

\begin{tabular}{|c|c|c|c|c|c|c|}
\hline \multirow{2}{*}{ Indexes } & \multicolumn{6}{|c|}{ For trousers with $E_{\mathrm{hg}}=8 \mathrm{~cm}$} \\
\hline & \multicolumn{2}{|c|}{ Fabric 1} & \multicolumn{2}{|c|}{ Fabric 2} & \multicolumn{2}{|c|}{ Fabric 3} \\
\hline$B_{\mathrm{wr} .}$ gf.cm2/cm; & \multicolumn{2}{|c|}{0.05} & \multicolumn{2}{|c|}{0.15} & \multicolumn{2}{|c|}{0.37} \\
\hline$B_{\text {wf. }}$ gf.cm2/cm; & \multicolumn{2}{|c|}{0.09} & \multicolumn{2}{|c|}{0.06} & \multicolumn{2}{|c|}{0.63} \\
\hline $\mathrm{G}_{\mathrm{wr}} . \mathrm{gf} / \mathrm{cm} \cdot \mathrm{deg}$ & \multicolumn{2}{|c|}{0.92} & \multicolumn{2}{|c|}{0.41} & \multicolumn{2}{|c|}{2.12} \\
\hline $\mathrm{G}_{\text {wf. }} \mathrm{gf} / \mathrm{cm}$.deg. & \multicolumn{2}{|c|}{0.98} & \multicolumn{2}{|c|}{0.44} & \multicolumn{2}{|c|}{2.63} \\
\hline \multicolumn{7}{|c|}{ Hip girth level. Wrinkle coefficients Cw \%. } \\
\hline & theoretical & real & theoretical & real & theoretical & real \\
\hline $\mathrm{CW}_{2} . \mathrm{CW}_{5}$ & 17.43 & 16.12 & 15.69 & 13.25 & 21.5 & 24.64 \\
\hline$C w_{3} . C w_{4}$ & 4.39 & 6.53 & 1.34 & 0.86 & 0.48 & 2.63 \\
\hline$C w_{1} \cdot C w_{6}$ & 12.51 & 10.76 & 13.25 & 14.74 & 10.94 & 13.58 \\
\hline \multicolumn{7}{|c|}{ Thigh girth level. Wrinkle coefficients $\mathrm{Cw} \%$. } \\
\hline & theoretical & real & theoretical & real & theoretical & real \\
\hline$C w_{3}$ & 5.32 & 7.42 & 1.55 & 3.65 & 20.19 & 18.62 \\
\hline$C w_{4}$ & 8.79 & 10.68 & 11.41 & 12.26 & 16.81 & 12.89 \\
\hline$C w_{5}$ & 8.36 & 5.23 & 7.65 & 4.86 & 25.81 & 22.63 \\
\hline$C w_{6}$ & 9.14 & 10.43 & 2.75 & 1.06 & 0.12 & 1.54 \\
\hline
\end{tabular}

Theoretical wrinkle coefficients values obtained by using new equations are correct and the error is about 3 to $12 \%$. This difference can be admitted because of the mobile structure of the clothes shell surface.

Theoretical cross-sections of "body-trousers" systems with $E_{\mathrm{hg}}=8 \mathrm{~cm}$ for three typical fabrics with different shell properties are shown on the figure 6

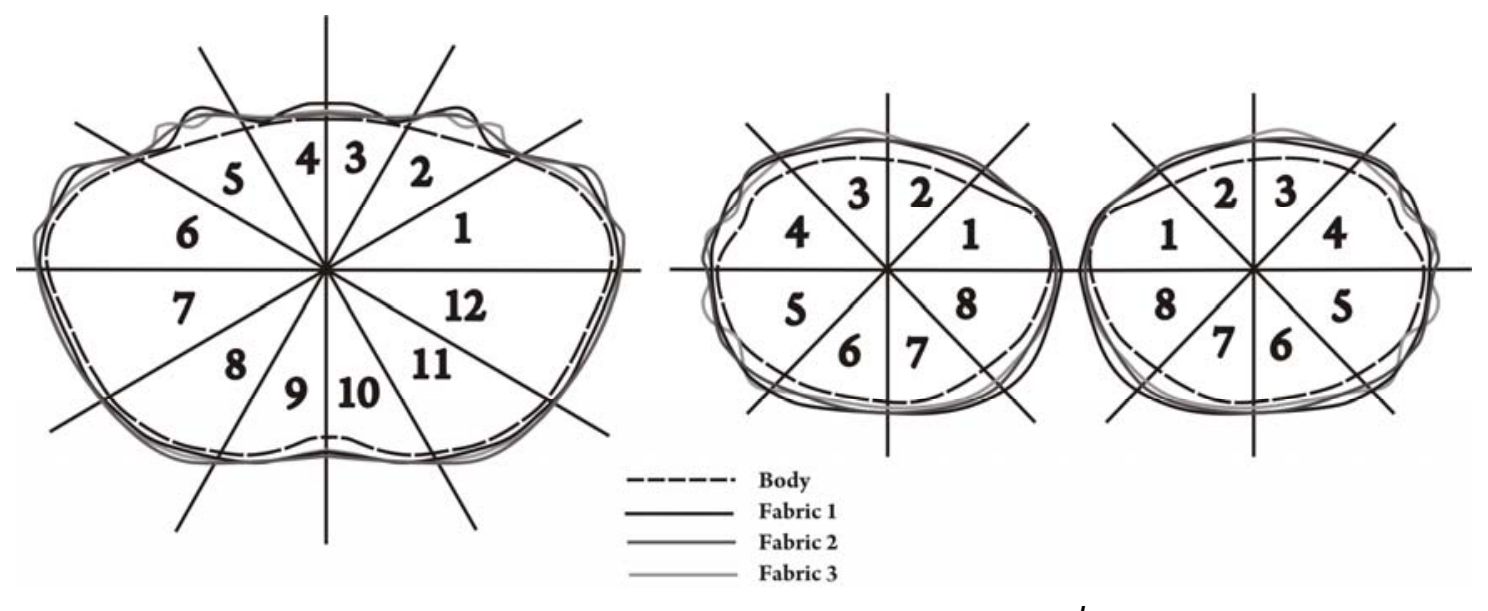

a

$b$

Fig. 6. Theoretical cross-sections of "body-trousers-fabric" systems with $E_{h g}=8 \mathrm{~cm}$ on hip girth (a) and thigh girth (b) levels.

The theoretical cross-sections can be used for comparing it to the real cross-sections of the scanned systems and for the identification of the fabrics shell properties.

Identification method includes the calculation of the wrinkle coefficients values using the equation (1) and comparing them with the theoretical values for required fabric with known indexes of bending rigidity $B$ and shear stiffness $G$.

Thus, the proposed mathematical equations allow to identify the indexes of fabric shell properties in the real "body-clothes" scanned systems and to improve design of the realistic surface of virtual clothes in accordance to the fabric properties and pattern parameters. 


\section{Conclusion}

Studying of the 3D clothes shell in the real "body-clothes" scanned systems has allowed to develop the new indexes of fabric shell properties and the wrinkle coefficient. The new indexes can describe realistic structure of the fabric surface with folds and wrinkles.

Relations between the wrinkle coefficient and the fabric indexes of bending rigidity and shear stiffness have been established and mathematical equations have been obtained.

Results of the research allow to identify the indexes of fabric shell properties in the real "body-clothes" scanned systems and to design realistic surface of "body-trousers" 3D systems with the required fabric properties.

\section{References}

1. Zhong, Y., Xu, B., (2009): "Three-dimensional Garment Dressing Simulation”. Textile Research Journal, vol. 79. pp. 792-803.

2. Chichocka, A., Bruniaux, P., Koncar, V., (2007): "Modelling of Virtual Garment Design in 3D", Research Journal of Textile and Apparel, vol. 11. No. 4. pp. 166-178.

3. F.Fathy.Saied, E., (2011): "Prediction of Garment Drapability Based on Fabric Properties", Journal of American Science, vol. 7, pp. 596-603.

4. Narahari, K., (2008): "Enhancing accuracy of drape simulation. Part I Investigation of drape variability via 3D scanning", Journal of the Textile Institute, vol.99, No. 3, pp 211-218.

5. Wang, P., Cheng, K., (2011): "Dynamic drape property evaluation of natural fiber woven fabrics using a novel automatic drape-measuring system", Textile Research J., vol.81. pp. 1406-1415.

6. Juan, F., Jiang, Y Z., Shan, Y W., (2011): "Sketch-based wrinkle generation for threedimensional virtual garment prototyping", Textile Research J,. vol. 81. pp. 1893-1902.

7. Park, SM, Nam, YJ and Choi, KM., (2004): "A basic study for the standardization of 3D anthropometry", Proceedings of Korean Society of Clothing and Textiles, pp.57.

8. Wang, J. and Lu, G., (2011): Customer participating 3D garment design for mass personalization. Textile Research Journal, vol.83, pp.187-204.

9. Sunyoon, Ch. and Ashdown, S., (2011): "3D body scan analysis of dimensional change in lower body measurements for active body positions”, Textile Research Journal, vol. 81. pp.81-93.

10. Petrova, A., Ashdown, S., (2008): "Three-Dimensional Body Scan Data Analysis. Body size and shape dependence of ease values for pantrs fit", Clothing and Textile Research Journal, No. 3, pp.62-71.

11. Zvereva, lu., Kuzmichev, V., Li J, (2011): "Trousers three-dimensional shapes simulation in the real system of «figure - trousers»", Journal Technology of Textile Industry, No. 5. pp. 83-88.

12. Kuzmichev, V., Gukova, I., Gnidenko, (2007): "Method of the bodies and clothes scanned systems analysis", Journal Technology of Textile Industry, No.1, pp.90-93.

13. Kawabata evaluation system (accessed 2011): www.tx.ncsu.edu/tpacc/comfortperformance/kawabata-evaluation-system.cfm 